Original article

\title{
MORPHOLOGICAL STUDIES OF THE CANINE HEPATIC PORTAL SYSTEM
}

\author{
G. I. GEORGIEV ${ }^{1}$, I. RAYCHEV ${ }^{2}$, N. MEHANDZHIYSKI ${ }^{2}$, L. HRISTAKIEV ${ }^{1}$, \\ G. D. GEORGIEV ${ }^{1} \&$ E. SAPUNDZHIEV ${ }^{1}$ \\ ${ }^{1}$ Department of Anatomy, Histology and Physiology; ${ }^{2}$ Department of \\ Surgery, Radiology, Obstetrics and Gynecology; University of Forestry, \\ Faculty of Veterinary Medicine, Sofia, Bulgaria
}

\section{Summary}

Georgiev, G. I., I. Raychev, N. Mehandzhiyski, L. Hristakiev, G. D. Georgiev \& E. Sapundzhiev, 2018. Morphological studies of the canine hepatic portal system. Bulg. J. Vet. Med., 21, No 4, 406-419.

The aim of the study was to track hepatic veins and their inflow into the caudal hollow vein using corrosion cast, contrast helical computed tomography imaging (CT), conventional ultrasonography (US) and contrast-enhanced ultrasonography (CE-US). The corrosion cast was made on a deceased dog's liver by injecting solidifying coloured plastic materials through the portal vein and caudal vena cava, enabling macroscopic visualisation of the portal and hepatic venous blood vessel branches up to the perilobular veins. Prior to the CT and US scans, the studied dogs were anaesthetised. The assessments were performed following administration of contrast media into the cephalic vein. The CT-scan images, together with the CT and CE-US images of the liver in the transversal and transversal-sagittal planes on the level from the eighth thoracic to the second lumbar vertebras, were compared. The canine hepatic portal system studied using corrosion cast, contrast imaging CT and US methods showed constant pattern of blood supply anatomical disposition. These approaches were used as a model for diagnosing portosystemic shunts in subsequent studies.

Key words: computed tomography, dog, portal and hepatic veins, ultrasonography

\section{INTRODUCTION}

The mammalian portal vein is formed by four large venous inlets - v. lienalis, v. gastroduodenalis, $v$. mesenterica cranialis and v. mesenterica caudalis (Frewein \& Habel, 2012, Evans \& de Lahunta, 2013; Vodenicharov, 2014). Portosystemic shunts (PSSs), which are anomalies in the development of the liver venous blood vessels, are sometimes observed in these functional sources. They are known as derivations of these large inlets or their smaller branches toward the caudal vena cava, where bypasses of the liver portal system are found. PSSs are intra- or extrahepatic depending on their location, single or multiple according to their 
structure, or, congenital or acquired in terms of their origin (Barrett et al., 1976; Andrew et al., 2005). The different types of PSSs have been researched more precisely and thoroughly in the human liver (Gallego et al., 2004; Soon et al., 2006).

An increased number of research reports on vascular abnormalities in liver blood supply of domestic animals has recently appeared. With the expansion of diagnostic imaging methods, other malformations of the portal vein in dogs of different breeds have been detected (Santilli \& Gerboni, 2003; Andrew et al., 2005; Nelson \& Nelson, 2011). The liver is the most common clinically affected organ, and vascular anomalies appear spontaneously along with encephalopathy. They have nonspecific symptoms, and differentiating them from other disorders of the nervous and digestive system is quite complicated (Yoon et al., 2014). While an extrahepatic portosystemic shunt may be suspected based on signalment, clinical signs and biochemical testing, imaging is required to make a definitive diagnosis and obtain the features of the shunting vessel's morphology (Nelson \& Nelson, 2011). With this regard, different types of portal shunts could be studied more comprehensively in dog veterinary practices (Barrett et al., 1976; Nelson \& Nelson, 2011). It is believed that extrahepatic PSSs in dogs originate from the gastrosplenic and gastroduodenal veins and terminate in the hepatic segment of the caudal vena cava. A splenophrenic shunt in a Shih Tzu was demonstrated by CT with volumerendered imaging, whereas a transverse CT image revealed that the large shunting vessel passed cranially to the liver along the diaphragm and entered the caudal vena cava from the left side. Large hepatic veins entered this shunt from its ventral aspect, near to the place where the shunt went into the caudal vena cava (Yoon et al., 2014). It is possible to visualise these structures in human and dog livers by contrast imaging methods, allowing further assessment of whether they can be surgically removed (Kenji et al., 2000; Yoon et al., 2014). An extrahepatic PSS in a mid-sized dog breed - English Cocker Spaniel - was demonstrated in a previous study. It was classified as congenital PSS with hypoplasia of the portal vein, which developed multiple collateral vessels as well as links with veins of the portal vessel system of splenic, ovarian and renal veins. A mesogonadal shunt was observed in the dog. In a large dog breed - Rhodesian Ridgeback -an extrahepatic PSS was observed again. In this case, there was a single shunt at the place where the middle colic vein bypasses the portal system. It was infused into the inlets of the caudal vena cava (Georgiev et al., 2015).

Cornillie (2008) reports additional abnormal hypoplasia of the portal vein together with pre-hepatic portocaval shunts in a Border Collie dog. Furthermore, stenosis of the caudal vena cava at liver level, accompanied by dilation caudally of the stenosis with several connections to the azygous vein, was reported in the dog (Hunt et al., 1998; Harder et al., 2002; Cornillie, 2008).

Congenitally, the portal vein may form a direct connection between the caudal vena cava and the hepatic veins. Referenced data also shows that similar 
PSSs can be observed during the perinatal period, though they rarely lead to disorders, such as interruption of the canine portal vein (Bertolini, 2010). In this regard, knowledge of the embryonic liver's blood supply enables identification and correct diagnosis of intrahepatic PSS in dogs (Hyttel et al., 2010).

As interventional radiography is increasingly used in the treatment of intrahepatic shunts in dogs, accurate pretreatment diagnosis is important. What is more, precise preoperative imaging of extrahepatic portosystemic shunts in dog may guide surgical interventions, reducing morbidity and the time taken to operate, as well as allowing accurate assessment of complex shunts (Santilli \& Gerboni, 2003; Nelson \& Nelson, 2011). Computed tomography (CT) of the liver with added contrast has been used for evaluation of hepatic vessels for liver transplantation, liver lobectomy, interventional radiology and diagnosis of hepatocellular carcinoma in humans (Oishi et al., 2015)

The gross anatomy of the portal and hepatic vein in the dog was reported through venous portography, corrosion cast and gross dissection by Kalt \& Stump (1993) and Hall et al. (2015).

Recently, the hepatic venous system was studied through corrosion casts and skeletonised by Ursic et al. (2014) and Mari \& Acocella (2015). The authors classified the hepatic veins in three main groups: the right hepatic veins of the caudate process and right lateral liver lobe, as the right section was perfused by the right portal branch and drained by independent hepatic veins; the middle hepatic veins of the right medial and quadrate lobes and the left hepatic veins of both left liver lobes and the papillary process as most of the left section, perfused by the left portal branch, and was drained by the main hepatic vein deriving from the middle and the left hepatic vein confluence (Ursic et al., 2014; Mari \& Acocella, 2015). These examinations showed that the number of hepatic veins was underestimated in the classic nomenclature (Schaller, 2007; Frewein \& Habel, 2012) and a new nomenclature may be less confounding in surgical settings (Mari \& Acocella, 2015)

Visualisation of hepatic vessels in Beagle dogs by contrast CT using triple phase images was evaluated as useful for diagnosis of liver failure and for creating a standardised description of the approaches to liver lobectomy in the dog (Oishi et al., 2015).

The systematic liver evaluation by ultrasonography was recorded to define in details the anatomy of the portal and hepatic veins in the dog (Wu \& Carlisle, 1995; Carlisle et al., 2005). Identification of the branches of both venous systems was complicated by the anatomical shape, the nutritional status and respiratory stage of the animals (Wu \& Carlisle, 1995; Carlisle et al., 2005). The same authors affirmed the importance to distinguish portal from hepatic veins and from veins of dilated bile ducts by better echogenicity of portal veins vs hepatic veins, which should be traced to their junction with caudal vena cava.

The objective of this study was to research intrahepatic segments and the formation of the portal vein and the hepatic veins, and their inflow into the caudal vena cava in clinically normal dogs using a corrosion cast, contrast computerised tomography, conventional and contrast enhanced ultrasonography, 
in an attempt to visualise these important venous blood vessels for potential diagnosis of vascular abnormalities of the canine liver portal system.

\section{MATERIALS AND METHODS}

\section{Animals}

This study was performed on 3 mature female mixed breed dogs. No respiratory or digestive abnormalities were found during the physical examination. The dogs weighed 15-20 kg and were clinically healthy. Two of the dogs were housed and treated in accordance with the rules approved by the local Ethics Committee (affiliated with the National Ethics Committee for Animal Safety, Welfare and Experimentation) pursuant to Ordinance 20 of $01 / 11 / 2012$, Section VI, Article 41, amended by the Bulgarian Ministry of Agriculture and Food supervised by the Bulgarian Food Safety Agency. One of the female dogs was in the $35^{\text {th }}$ day of pregnancy. The two animals recovered without complications after the CT and CE-US scans; the pregnant dog gave birth to healthy puppies without complications. The third female dog was euthanised for reasons unrelated to the study.

\section{Conventional and contrast-enhanced ultrasonography study}

The dogs were not given food for 6 hours and were then anaesthetised as followed: premedication with atropine sulfate (Sopharma-Bulgaria) - 0.02-0.04 mg/kg S.C.; induction with xylazine $2 \%$ (Alfasan, The Netherlands) at 0.5-1.5 $\mathrm{mg} / \mathrm{kg}$ I.M.; maintenance with ketamine $10 \%$ (Ketaminol 10\%, Intervet, The Netherlands) - $10 \mathrm{mg} / \mathrm{kg}$ I.V. (Thurmon et al., 1996; Mehandzhiyski et al., 2007).
The hair coat on the cranial abdomen was clipped. After gel application (Eco Gel 200), echography of this area was performed (Mindray DP-10 with a linear transducer and frequency 6.5-8.0 $\mathrm{MHz}$ ). The examinations were made in ventrodorsal or lateral views in transversal, longitudinal and transverselongitudinal planes.

Contrast-enhanced ultrasonography was performed on similarly prepared dogs. Contrast medium (25 mg Sonovue ${ }$, Bracco International B. V., The Netherlands) was infused intravenously into the cephalic vein, at a dose of 1-2 $\mathrm{mL}$ per dog (Troianos et al., 2011). The solution was administered according to the manufacturer's recommendations. The US scan images acquired by both modes of echography were compared with CT-scan images.

\section{Computed tomography study}

The anaesthetised dogs were placed in sternal recumbency on the CT table. A contrast medium, sodium diatrizoate $76 \%$ (Urografin ${ }^{\circledR}$; $\quad$ Schering, Berlin, Germany), was administered by inserting a Luer Lock IV syringe into the cephalic vein at a dose of $20 \mathrm{~mL}$ per dog (Möller \& Reif, 2006; Troianos et al., 2011). CT was performed along the transversal planes from the eighth thoracic to the second lumbar vertebra 30-50 s after contrast application, in $1.5 \mathrm{~mm}$ thick helical CT slices at $5 \mathrm{~mm}$ intervals, by a Picker ${ }^{\circledR}$ CT PQ 5000 scanner. The CT scan images were analysed with computer software DIKOM-VIEWER and were subsequently compared with US and CEUS scan images and a corrosion cast.

Anatomical study - corrosion cast

After the US, CE-US and CT 


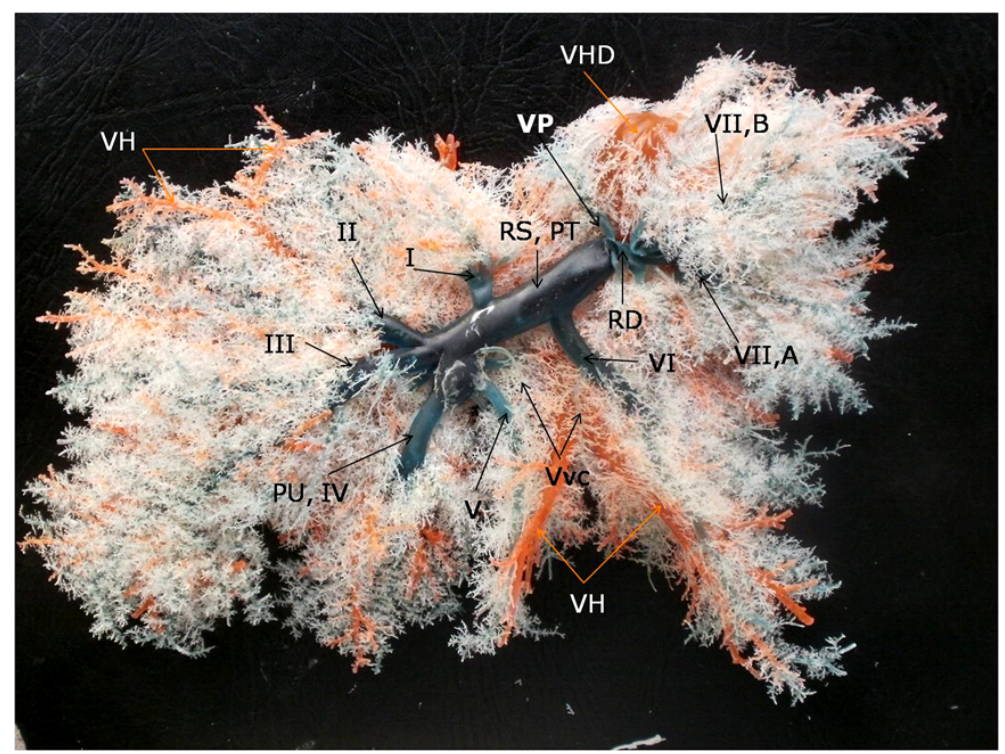

Fig. 1. Venous blood vessels of the dog liver, corrosion cast, visceral surface, segments according to Morozovoy. VP - portal ven; VHD - right hepatic vein; VH - hepatic vein; RS, PT - left branch, transverse part; RD - right branch; I, II, III, V, VI, VII,A, VII,B - segments, PU, IV - segment, umbilical part; $\mathrm{Vvc}$ - cystic veins.

examinations, the third female dog was euthanised for reasons unrelated to this study. Colour solutions of Duracril-Plus ${ }^{\circledR}$ (Spofa-Dental, Czech Rep.), respectively blue and red, were introduced into the liver portal vein and the caudal vena cava of the dog cadaver. The solutions were prepared by mixing dust and a solidifying substance in a ratio of $3: 1$, respectively. After full polymerisation, the canine liver was immersed into a 1:1 solution of sulfuric acid and tap water until complete corrosion of the soft tissues (Georgiev, 2012). The vessels of casts were identified and correlated to the analogous structures on the corresponding CT slices and US images according to Nomina Anatomica Veterinaria (Frewein \& Habel, 2012).

\section{RESULTS}

The hepatic portal system and tissue structure of the three healthy dogs could be clearly visualised by CT and US imaging and a contrast medium in the cephalic vein. Clinically relevant abdominal anatomic structures were identified and labelled for each CT, US, CE-US image. Some CT transverse slices were accompanied by dorsoventral radiographic images in which the lines depict places of $\mathrm{CT}$ imaging along transverse planes. Those multiple sections were selected from different dogs and were presented in a cranial to caudal progression from the level of the eighth thoracic vertebra to the level of the thirteenth vertebra.

Fig. 1 shows segments of the portal vein according to Morozovoy and hepatic veins on the visceral surface of the corrosion cast. Blue-coloured longer and larger left branch and shorter right 


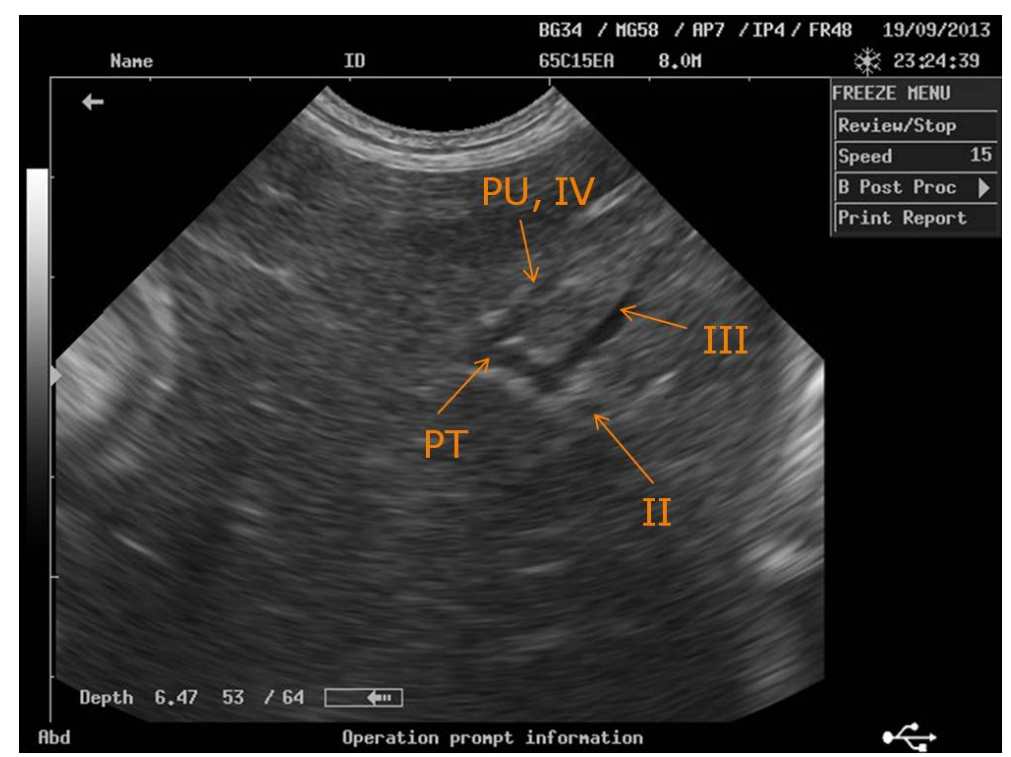

Fig. 2. Ventrodorsal transverse-longitudinal CE-US image through the middle part of the eighth thoracic vertebra. VH - hepatic vein; II - $2^{\text {nd }}$ segment; III - $3^{\text {rd }}$ segment; RS, PT - left branch, transverse part; PU, IV - left branch, umbilical part, $4^{\text {th }}$ segment.

branch of the portal vein were observed. The left branch (ramus sinister) was not directly joined to the intrahepatic part. It was separated in two main parts umbilical and transverse. The first part was directed sagittally toward the medial left hepatic lobe and both sides of fissura ligamentum teretis. The second originating part was located transversely. Six segments were supplied by the left branch, including the medial right hepatic lobe. The seventh segment came from the right branch, which was directly connected to the intrahepatic part and supplied the liver caudate process dorsally and the lateral hepatic lobe ventrally. Reddish-coloured hepatic veins and the left, mediate and right hepatic veins which join the caudal vena cava can be seen in the diagram of the surface of the corrosion cast. Cystic veins which drained blood from the gallbladder (Fig. 1) were identified on the visceral surface.

Below the vertebral level, the abdominal aorta, caudal lobes of the left and right lung, as well as arterial and venous vessels supplying them, were observed on the level of the eighth thoracic vertebra in the $\mathrm{CT}$ images. The caudal vena cava passes caudally under the right lung. The second and third segment were supplied and continued from the transverse part. They enter dorsally and ventrally into the lateral left hepatic lobe. The fourth segment supplies the medial left hepatic lobe which is directed toward fissura teres hepatis. The fifth segment is connected to the quadrate lobe medially of the gallbladder. Both segments branch off from the umbilical part of the portal vein. All these segments and their separation from the left branch of the portal vein were compared with US 
and CE-US scans (Fig. 2). The walls of these venous vessels had a hyperechoic elongated projection at longitudinal view and an oval shape at transverse projection compared with the relative hypoechoic hepatic parenchyma echotexture. The portal vein blood vessels had anechoic lumens, while hepatic veins with hypoechoic walls were observed (Fig. 2).

On the level of the ninth thoracic vertebra, the CT and CE-US slices continued ventrally along the abdominal aorta, caudal vena cava and hepatic vein on the dorsal margin of the liver, as well as the separation of the left branch of the transverse and umbilical part (Fig. 3). The flow from the left, middle and right hepatic veins or more accurately described as middle right hepatic vein into the caudal vena cava and smaller hepatic veins was visible on the CT scans, as well as the stomach wall, gallbladder and its cystic duct (Fig. 3).

On the level of the tenth thoracic vertebra and costovertebral joint, the $\mathrm{CT}$, US and CE-US images identified the undivided left branch, its first segment branching into the papillary hepatic process and its sixth segment on the right of the gallbladder (Fig. 4). Medially of the hyperechoic stomach wall and laterally of the anechoic gallbladder, the same segments were visualised ultrasonographically. The terminal part of the long third segment into the lateral left hepatic lobe near its acute margin were observed on the $\mathrm{CT}$ slices. 


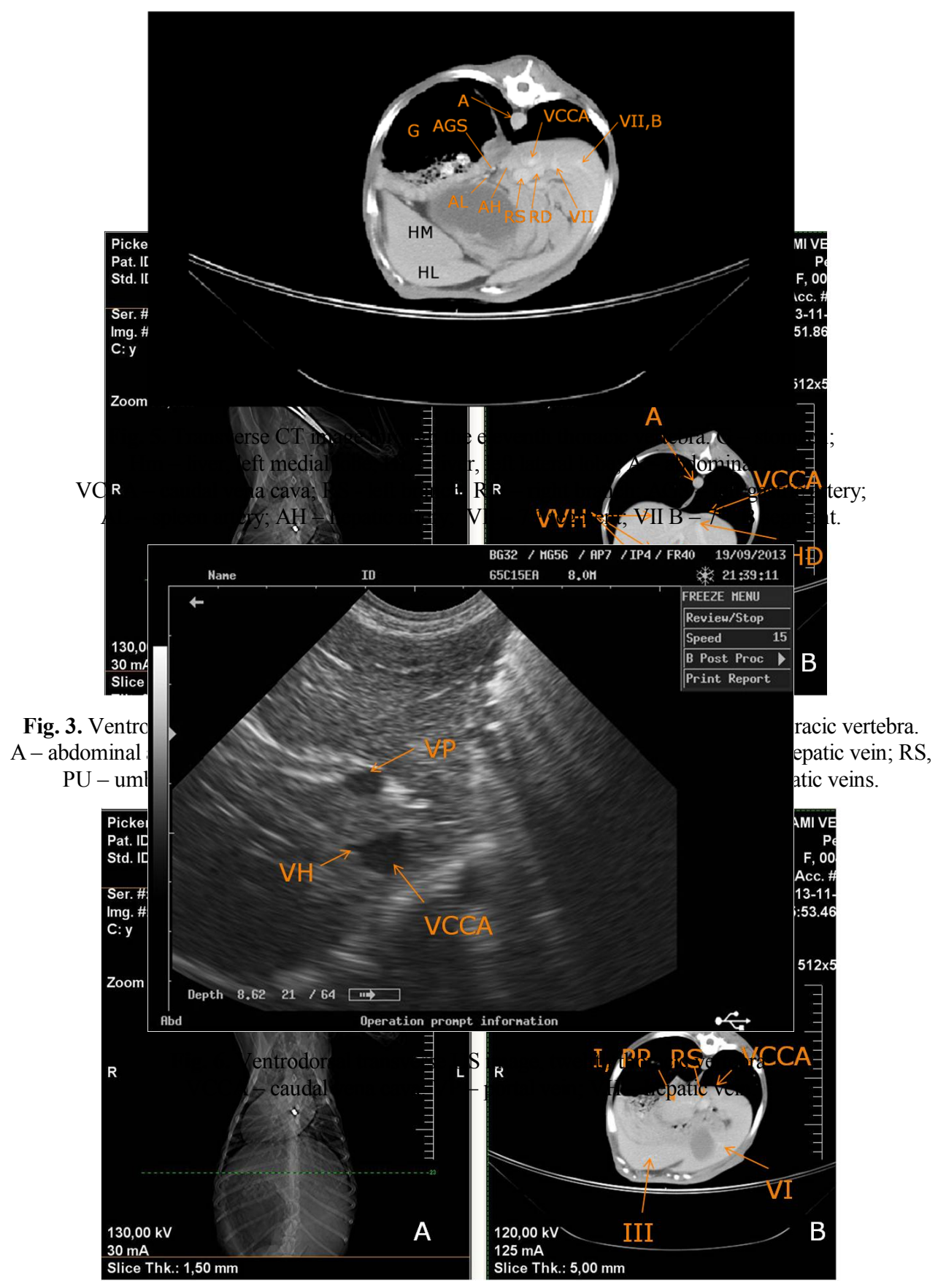

Fig. 4. Ventrodorsal radiograph (A) and transverse $\mathrm{CT}$ image (B) through the tenth costovertebral joint. VCCA - caudal vena cava; RS - left branch; I, PP - branches of $1^{\text {st }}$ segment into papillary process; III $3^{\text {rd }}$ segment; VI $-6^{\text {th }}$ segment. 
Morphological studies of the canine hepatic portal system

On the level of the eleventh thoracic vertebra, the CT, US and CE-US scans

were correlated to separation of the portal vein from its main blood vessels and branching from the $7^{\text {th }}$ segment into the right side of liver. Left gastric, spleen and hepatic arteries (Fig. 5) were identified on the CT slices. 
G. I. Georgiev, I. Raychev, N. Mehandzhiyski, L. Hristakiev, G. D. Georgiev \& E. Sapundzhiev

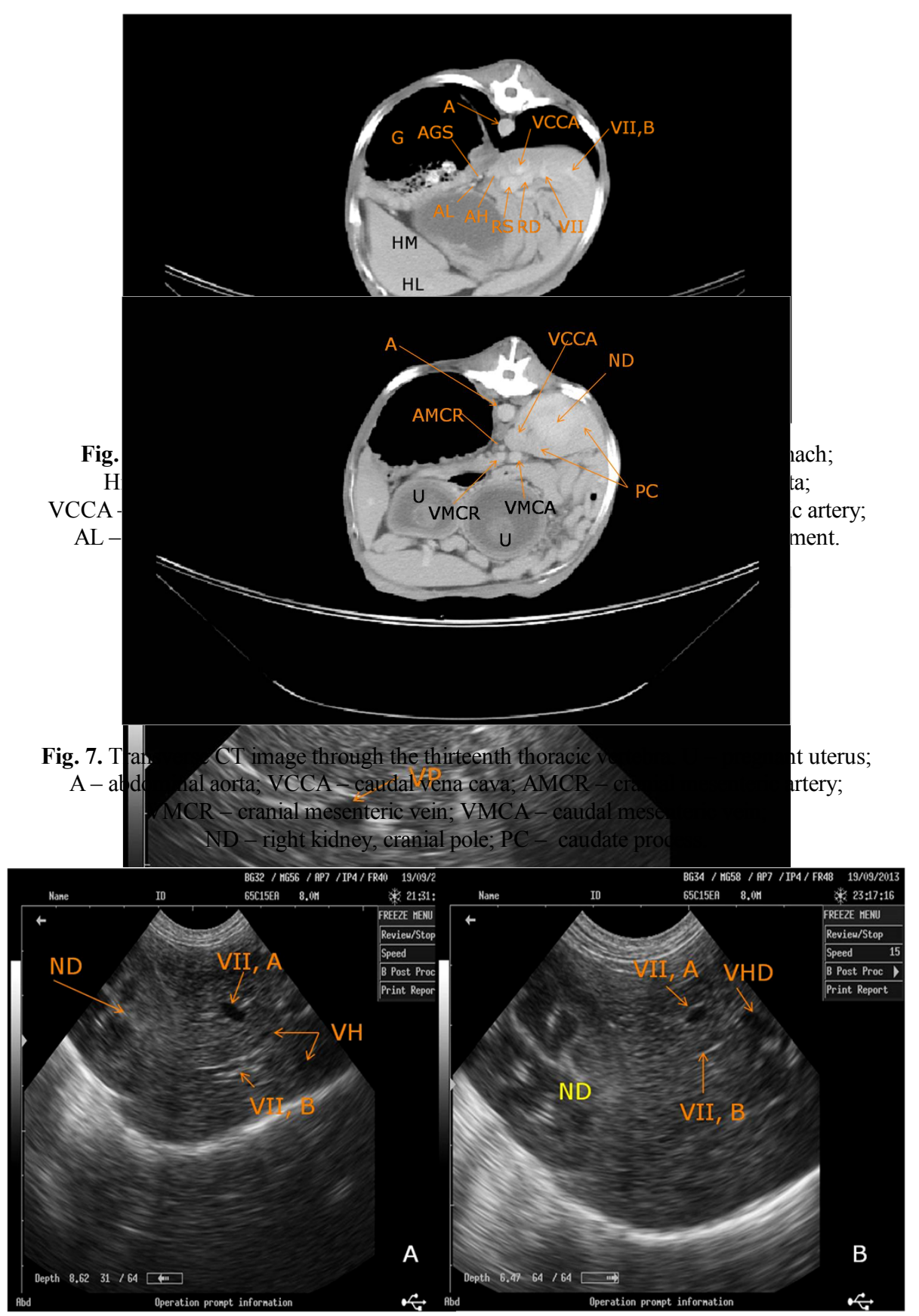

Fig. 8. Ventrodorsal longitudinal US image (A) and ventrodorsal longitudinal CE-US image (B) through the thirteenth thoracic vertebra. ND - right kidney; VII a $-7^{\text {th }}$ A-segment; $\mathrm{VII} b-7^{\text {th }} \mathrm{B}$-segment; $\mathrm{VH}-$ hepatic vein. 
On the level of the twelfth vertebra, the CT and US slices showed the formed portal vein branching from the $7^{\text {th }} \mathrm{B}$-segment into the caudate hepatic process. The cranial mesenteric artery above the portal vein, the stomach's left side, the spleen with its vein near the left abdominal wall and pregnant uterus horns ventrally were identified on the CT scan. The transverse sonogram visualised the caudal vena cava and hepatic vein's inflow into it (Fig. 6).

On the level of the thirteenth vertebra, the CT image examined the continuing caudal vena cava, cranial and caudal mesenteric vein before their confluence

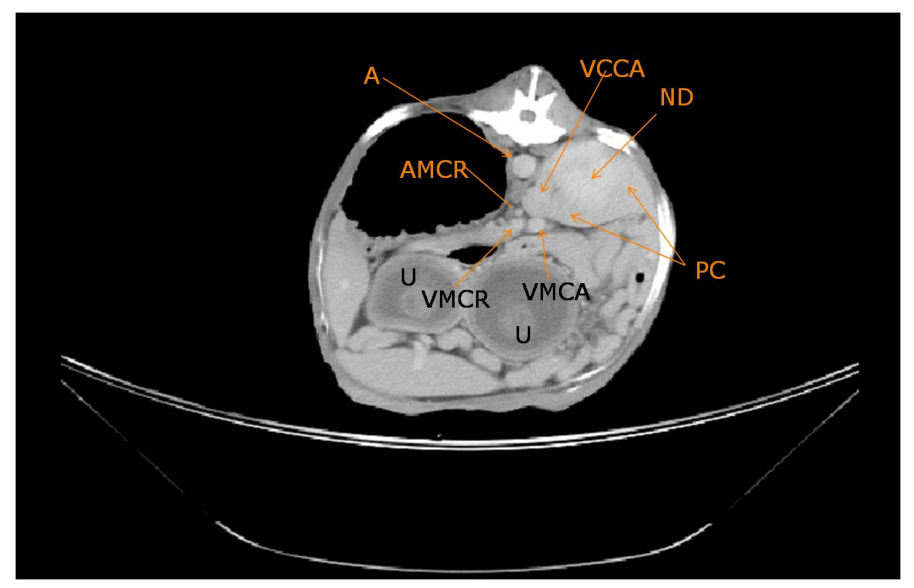

Fig. 7. Transverse $C T$ image through the thirteenth thoracic vertebra. $U$ - pregnant uterus; A - abdominal aorta; VCCA - caudal vena cava; AMCR - cranial mesenteric artery; VMCR - cranial mesenteric vein; VMCA - caudal mesenteric vein; $\mathrm{ND}$ - right kidney, cranial pole; $\mathrm{PC}$ - caudate process.

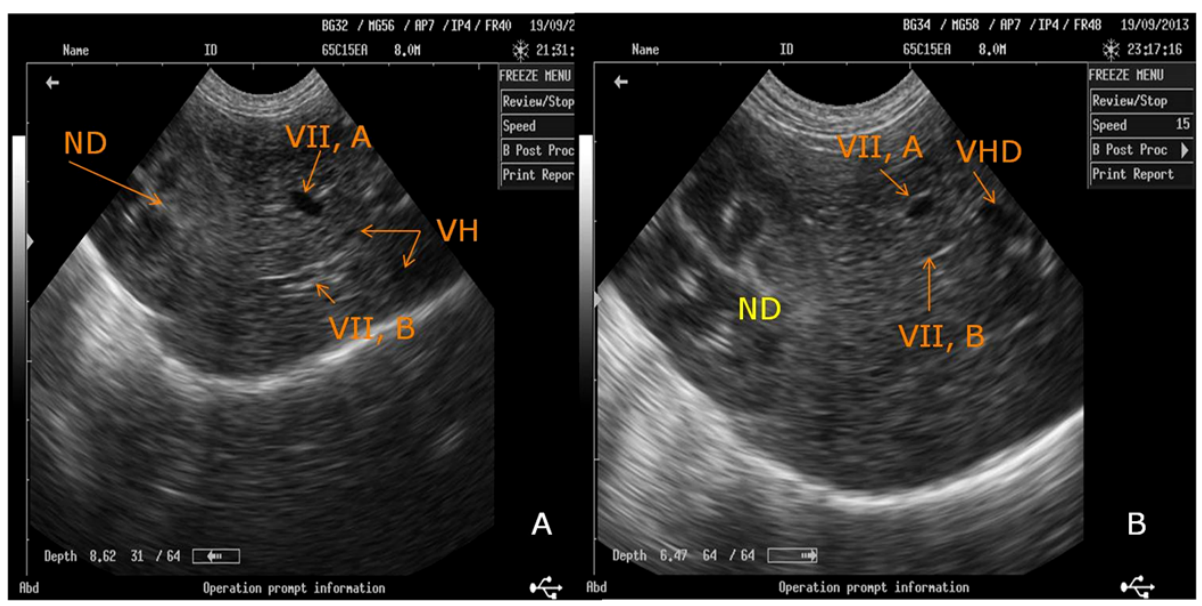

Fig. 8. Ventrodorsal longitudinal US image (A) and ventrodorsal longitudinal CE-US image (B) through the thirteenth thoracic vertebra. ND - right kidney; VII a $-7^{\text {th }}$ A-segment; $\mathrm{VII} b-7^{\text {th }} \mathrm{B}$-segment; VH - hepatic vein. 
into the portal vein, cranial mesenteric artery dorsally, caudate process and cranial pole of the right kidney dorsolaterally on the right side and pregnant uterus horns ventrally (Fig. 7). The structure of the right kidney and again the caudal vena cava and the hepatic vein's inflow into its longitudinal axis were observed on the CE-US slice.

As shown on Fig. 8, on the level of the thirteenth thoracic vertebra, the right kidney, the right parts of the liver, along with the segmentation of the portal vein and hepatic veins were compared before and after the introduction of echographic contrast medium. Normal kidney cortex and liver parenchyma with isoechoic structure are visible on Fig. 8A. After injection in the same organs, a hyperisoechoic structure was observed (Fig. 8B). After an injection in the same organs, a hyperisoechoic structure was observed on the right.

\section{DISCUSSION}

Details on the intrahepatic branching of the portal vein into five segments from the left branch, but into two segments sixth and seventh coming from the right branch according to Morozovoy - were reported in a human corrosion cast by Sinelynikov \& Sinelynikov (1992). Based on similarities between canine lobes and human segments a new nomenclature may be less confounding in surgical settings (Mari \& Acocella, 2015). The same branching was confirmed in sheep by corrosion cast, computed tomography and ultrasonography in another study of ours (Georgiev et al., 2012). The same segmentation of the portal vein was examined in dogs by Ursic et al. (2007).
This ramification of portal vein was quite similar to reports in some dogs. Our study however showed that the left branch has six segments which supply the central lobes, including the medial left and right hepatic lobe of the liver, while the right branch - only one seventh segment, which supplies the caudate process and the lateral right lobe of the liver (Fig. 1), corresponding to the report of Schaller (2007) and of Kalt \& Stump (1993). The left branch is stronger and separates from the transverse part, and passes transversely in the dog but ventrally in ruminants, while the umbilical part, located sagittaly in the $\mathrm{dog}$, is in the transversal plane in ruminants (Schaller, 2007; Vodenicharov, 2014). According to these authors, the last part led to the fissura teres hepatis, which formed the fourth intrahepatic segment, as shown in our corrosion cast, CT and US scans (Fig. 13). This intrahepatic part was the remainder of the umbilical vein and was connected by a venous duct to the caudal vena cava during the foetal period (Schaller, 2007; Hyttel et al., 2010; Vodenicharov, 2014).

The organs, structures and main vessels - abdominal aorta, caudal vena cava and portal vein into the cranial abdomen of the three dogs - were examined in the CT images between the ninth and tenth, eleventh and twelfth thoracic vertebra, between the T13 and first lumbar vertebra, but without details of branching (Rivero et al., 2009). The same vessels, as well as the right renal and hepatic vein, left gastric and hepatic artery were described by these authors only in macroscopic cross sections. The right branch of the portal vein, left and right gastric artery and vein, spleen artery and 
vein, hepatic artery and veins, branches of the cranial mesenteric artery and vein into the cranial abdomen of dogs were visualised by Done et al. (2009), but again only in transverse sections through the ninth, thirteenth thoracic and first lumbar vertebrae. These arterial and venous blood vessels into the cranial abdomen of dogs were identified on the CT images in the present study (Figs. 5, 7). The portal vein is formed by the confluence of 4 veins. Two of them are the cranial and caudal mesenteric vein (Kalt \& Stump, 1993; Evans \& de Lahunta, 2013; Schaller, 2007; Vodenicharov, 2014), seen on CT scan images on the level of the last thoracic vertebra of the $\operatorname{dog}$ (Fig. 7). The same authors described the diameter of the portal vein as being approximately $1.2 \mathrm{~cm}$ (Evans \& de Lahunta, 2013), and the hepatic veins and their inflow into the caudal vena cava were confirmed on the CT and US slices (Figs. 3, 6).

According to Hall et al. (2015) there was a broadly consistent pattern in the hepatic and portal supply to the individual liver lobes. The branching pattern of the major portal vein and hepatic vein in their study agrees to a certain extent with the present study. Corrosion casting can visualise tiny vessels better than $\mathrm{CT}$ as reported by some authors (Ursic et al., 2007; Hall et al. 2015). However, the diameter and position of hepatic vessels may differ in live dogs according to Oishi et al. (2015) so their report tried to define hepatic vessels using CT in normal, living dogs. As a result, it was possible to visualise better small vessels using CT imaging than the corrosion casting technique as well as using CT imaging corresponding to both conventional and contrast enhanced ultrasonography. It was found that the hepatic artery differed between each individual dog, whereas the portal vein and hepatic veins were constant (Oishi et al., 2015); this statement was also confirmed by us.

The number of various-sized hepatic veins of the right liver division ranged from 3 to 5 and included 1 to 4 veins from the caudate process and 2 to 4 veins from the right lateral liver lobe (Ursic et al., 2014) and a proper right hepatic vein draining blood from more than one lobe was never observed (Mari \& Acocella, 2015). One middle-sized vein from the left part of the right medial lobe running to the left and joining the collecting vein from the quadrate lobe, emptying separately in the caudal vena cava, was reported by above mentioned authors. Designated as middle right hepatic vein (v. hepatica media dextra) by Ursic et al., (2014) it was visualised in our study on the CT-images (Fig. 3). The other vein a large-sized vein from the remainder of the central division, which frequently joined the common left hepatic vein from the left liver lobes (Ursic et al., 2014) deriving from the middle and the left hepatic vein confluence (Mari \& Acocella, 2015) was confirmed on the same CT images (Fig. 3). The caudate process of the caudate lobe, which drained directly into the caudal vena cava, was traced by us on the ultrasonographic slices.

The systematic evaluation of the liver by ultrasonography found a constant pattern of venous branching to each lobe of the canine liver with little interindividual variations. All liver lobes contained definite venous branches so that the left lateral and medial, quadrate, right medial and lateral, caudate and 
papillary veins could be distinguished in each venous system $(\mathrm{Wu} \&$ Carlisle, 1995; Carlisle et al., 2005) confirmed through conventional and contrast enhanced untrasongraphy in this study (Fig. 2, 8). The right lateral and caudate hepatic veins were identified more easily from the right side with the transducer positioned between the ninth and eleventh intercostals space but all other vessels from each venous system were examined with ventrally positioned transducer which was borrowed from the ultrasonographic study of $\mathrm{Wu} \&$ Carlisle (1995).

Details of ultrasonographic structures of the kidneys, liver, portal and hepatic veins, isoechoic cortex of kidneys and liver, hyperechoic walls of portal vein elongated on the longitudinal and oval on the transverse projections, hypoechoic walls and anechoic lumen of hepatic veins were reported by different authors (Wu \& Carlisle, 1995; Burk \& Feeney, 2003; Mannon, 2006; Penninck \& d'Anjou, 2008). In this study, the echotexture of venous blood vessels and branching of the portal vein in the canine liver were confirmed. Also, it was demonstrated that the cortex of right kidney, venous vessels and structure of the liver became hyperisoechoic after being injected with a contrast medium (Fig. 8A, B).

\section{CONCLUSION}

The results of our study indicate that vascular injection is useful in revealing anatomic structures and arterial and venous vessels on CT and US images. These methods provide detailed information about the branching and formation of the portal vein and the inflow of the hepatic veins into the caudal vena cava in canine livers. Radiologists could use such information in contrastenhanced diagnostic imaging techniques (ultrasound, computed tomography and magnetic resonance images). This research could be useful as a reference for evaluating CT and US images of abnormalities of the hepatic portal system and for quick diagnosis of these shunt venous vessels in dogs.

\section{REFERENCES}

Andrew, E. K., 2005. Portosystemic Shunts. Textbook of Veterinary Internal Medicine. Ettinger and Feldman.

Barrett, R. E., A. De Lahunta \& W. J. Roenick, 1976. Four cases of congenital portacaval shunt in the dog. Journal of Small Animal Practice, 17, 71-85.

Bertolini, G., 2010. Imaging anatomy of congenital extrahepatic portosystemic shunt in dogs. In: Proceedings of the XXVIII ${ }^{\text {th }}$ Congress of the European Association of Veterinary Anatomists, July 2010, Paris, France.

Burk, R. L. \& D. A. Feeney, 2003. Small Animal Radiology and Ultrasonography: A Diagnostic Atlas and Text. Saunders and Elseiver.

Carlisle, C. H., J. X. Wu, \& J. Trevor, 2005. Anatomy of the portal and hepatic veins of the dog: A basis for systematic evaluation of the liver by ultrasonography. Veterinary Radiology and Ultrasound, 36, 227-233.

Cornillie, P., 2008. Development of the caudal vena cava in the pig embryo: A threedimensional angiogenic model. $\mathrm{PhD}$. thesis. Gent University, Gent.

Done, S. H., P. C. Goody, S. A. Evans \& N. C. Stickland, 2009. Color Atlas of Veterinary Anatomy: The Dog and Cat, vol 3, Mosby Int.

Evans, H. E. \& A. De Lahunta, 2013. Miller's 
Anatomy of the Dog, $4^{\text {th }}$ edn, Saunders and Elsevier.

Frewein, J. \& R. Habel, 2012. Angiology, Veins. In: Nomina Anatomica Veterinaria, $5^{\text {th }}$ edn (revised version). Hannover (Germany), Columbia, MO (U.S.A.), Ghent (Belgium), Sapporo (Japan), published by the Editorial Committee.

Gallego, C., M. Miralles \& M. Marín, 2004. Congenital hepatic shunts. Radiographics, 24, 755-772.

Georgiev, G. I., I. Raychev, G. D. Georgiev, N. Mehandzhiyski \& L. Hristakiev, 2012. Morphologic study on the portal vessels system in sheep's liver. In: Procedings of the Conference Tradition and Modernity in Veterinary Medicine, Sofia, Bulgaria, pp. 87-97 (BG).

Georgiev, G. I., M. Stefanova \& V. Manov, 2015. Two types of portosystemic shunts of the $\operatorname{dog}$ - a clinical case. MedInform, 2, 175-183.

Hall, J. L., P. Mannion \& J. F. Ladlow, 2015. Canine intrahepatic vasculature: Is a functional anatomic model relevant to the dog? Veterinary Surgery, 44, 27-34.

Harder, M. A., D. Fowler, J. W. Pharr, K. A. Tryon \& C. Shmon, 2002. Segmental aplasia of the caudal vena cava in a dog. Canadian Veternary Journal, 43, 365-368.

Hunt, G. B., C. R. Bellenger, R. Borg, K. Ruth, P. L. Youmans, C. Tisdall \& R. Malik, 1998. Congenital interruption of the portal vein and caudal vena cava in dogs: Six case reports and a review of the literature. Veterinary Surgery, 27, 203-217.

Hyttel, P., F. Synowatz, M. Vejlsted \& K. Betteridge, 2010. Essentials of Domestic Animal Embryology. Saunders \& Elsevier.

Kalt, D. J. \& J. E. Stump, 1993. Gross anatomy of the canine portal veins. Anatomy Histology Embryology, 22, 191-197.

Kenji, I., T. Toshitaka, M. Koichi \& I. Yoshihiro, 2000. Transhepatic portosystemic shunts: CT appearance and anatomic correlation. American Journal of Roentgenology, 175, 153-157.
Mannion, P., 2006. Diagnostic Ultrasound in Small Animal practice. Blackwell Publishing Company.

Mari, L. \& F. Acocella, 2015. Vascular anatomy of canine hepatic venous system: a basis for liver surgery. Anatomy Histology Embryology, 44, 212-224.

Mehandzhiyski, N., Z. Baychev \& B. Aminkov, 2007. Effect of xylazine - ketamine anesthesia on hormonal distress during surgery. Experimental Pathology and Parasitology, 10, 9-13.

Möller, T. \& E. Reif, 2006. Pocket Atlas of Radiographic Anatomy, Thieme Flexibooks.

Nelson, N. C. \& L. L. Nelson, 2011. Anatomy of extrahepatic portosystemic shunts in dogs as determined by computed tomography angiography. Veternary Radiology and Ultrasound, 52, 498-506.

Penninck, D. \& M. A. D'Anjou, 2008. Atlas of Small Animal Ultrasonography. Blackwell Publishing Company.

Rivero, M. A., J. M. Vázquez, F. Gil, J. A. Ramírez, J. M. Vilaz, A. De Miuel \& A. Arencibia, 2009. CT-soft tissue window of the cranial abdomen in clinically normal dog. Anatomy Histology Embryology, 38, 18-22.

Oishi, Y., K. Tani, H. Nakazawa, K. Itamoto, T. Haraguchi \& Y. Taura, 2015. Anatomical evaluation of hepatic vascular system in healthy beagles using X-rays contrast computed tomography. The Journal of Veterinary Medical Science, 77, 925-929.

Santilli, R. A. \& G. Gerboni, 2003. Diagnostic imaging of congenital porto-systemic shunts in dogs and cats: A review. The Veterinary Journal, 166, 7-18.

Schaller, O., 2007. Angiology, Veins. In: Illustrated Veterinary Anatomical Nomenclature, $2^{\text {nd }}$ edn, Ferdinand Enke Verlag, Stuttgart, pp. 374-375.

Sinelynikov, R. D. \& Y. R. Sinelynikov, 1992. Atlas of Human Anatomy, vol. 3. Medicine, Moskow.

Soon, M. S., Y. Y. Chen \& H. H. Yen, 2006. Spontaneous intrahepatic portosystemic 
venous shunt. Journal of Gastroenterology and Hepatology, 21, 918

Thurmon, J. C., W. J. Tranqualli \& G. J. Benson, 1996. Veterinary Anesthesia, $3^{\text {rd }}$ edn, Williams and Wilkins.

Troianos, C. A., G. S. Hartman, K. E. Glas, N. J. Skubas, R. T. Eberhardt, J. D. Walker \& S. T. Reeves, 2011. Guidelines for performing ultrasound guided vascular cannulation. Journal of the American Society of Echocardiography, 24, 1291-318.

Uršič, M., D. Ravnik \& M. Hribernik, 2007. Gross anatomy of the portal vein and hepatic artery ramifications in dogs: Corrosion study. Anatomy Histology Embryology, 6, 83-87.

Uršič, M., M. Vrecl \& G. Fazarinc, 2014 Corrosion cast study of the canine hepatic veins. Folia morphologia, 73, 475-481.

Vodenicharov, A., 2014. Angiology. In: Anatomy of the Domestic Animals, vol 3, $3^{\text {rd }}$ edn, ed. G. Kovachev, G. D. Georgiev \& A. Vodenicharov, Kota Publishing Bulgaria, pp. 151-154 (BG).

Wu, J. X. \& C. H. Carlisle, 1995. Ultrasonographic examination of the canine liver based of recognition of the hepatic and portal veins. Veterinary Radiology and Ultrasound, 36, 234-239.

Yoon, H., M. Roh \& S. Jeong, 2014. Surgical correction of splenophrenic shunt in a dog: A case report. Veterinarni Medicina, 59, 396-402.

Paper received 04.11.2016; accepted for publication 06.02.2017

\section{Correspondence:}

Dr. Georgi I. Georgiev, DVM, PhD

Department of Anatomy, Histology and

Physiology

Faculty of Veterinary Medicine,

University of Forestry, Sofia Bulgaria tel: +359898743055

e-mail: dr_gigeorgiev@abv.bg 